\title{
Gesundheitskarte: eine Drehscheibe für Gesundheitsnetze
}

\author{
I. Cassis, M. Della Santa
}

Projekt «Rete sanitaria» (www.retesan.net), Kantonsärztlicher Dienst, Gesundheits- und Sozialdepartement, Bellinzona

1 Sorgenbarometer 2001. GfS, Bern, 2001.

2 Gesetz vom 18. März 1994, in Kraft ab 1. Januar 1996.

3 Bericht des Kantonsrates TI zu den Richtlinien und zum Finanzplan 1996-1999. Regierungsrat, Oktober 1995.

4 Bericht des Kantonsrates TI zur dritten Nachführung der Richtlinien und des Finanzplanes 1996-1999. Regierungsrat, November 1998 (S. 20).

Korrespondenz:

Dr. med. Ignazio Cassis, MPH

Medico cantonale

Via Dogana 16

CH-6501 Bellinzona
Die Gesundheit ist für die Schweizer Bevölkerung ein wichtiges Anliegen [1]. Obschon die meisten, auch unter den politischen Vertretern, grundsätzlich mit dem Gesundheitssystem zufrieden sind, ist dessen Stabilität doch infolge des kontinuierlichen Anstiegs der Gesundheitskosten immer ernsthafter in Frage gestellt. Um aber ein qualitativ so hochstehendes System aufrechterhalten zu können, ist es unentbehrlich, dessen Wirksamkeit, Effizienz und Gerechtigkeit in ein Gleichgewicht zu setzen. Wirksam indem es die erwünschten Ziele zu erreichen vermag, effizient im Sinne einer optimalen Verteilung der verfügbaren Ressourcen (Kosten-Nutzen-Verhältnis) und gerecht, indem es das Recht auf Behandlung aller (Gleichberechtigung) sowie die Anerkennung der jeweils besten Behandlung eines jeden (Gerechtigkeit) gewährleistet.

Bereits Mitte der neunziger Jahre begann der Tessiner Regierungsrat (RR) - parallel zum eidgenössischen Notfallsrecht zur Kontrolle der Gesundheitskosten und zu den KVG-Vorbereitungen [2] - nach konkreten Möglichkeiten zu suchen, um der Kostenentwicklung im Gesundheitswesen entgegenwirken zu können. Im Oktober 1995, im Rahmen des Grundziels «Schutz vor dem Ausschluss», gab der RR der Notwendigkeit Ausdruck, die stationäre, halbstationäre, ambulante und Spitex-Betreuung besser koordinieren zu wollen, um den Patienten eine optimale Therapie anbieten zu können [3]. Das heisst mit anderen Worten: tiefere Kosten bei gleichem Resultat.

Ein Gesundheitssystem ist eine komplexe und multifunktionelle Einrichtung. Sie setzt sich aus einer Vielfalt von Akteuren der verschiedensten Kompetenzbereiche zusammen, deren spezifische Interessen sich zwar strukturell ergänzen, konjunkturell aber oft gespannt gegenüberstehen. Nicht immer ist es ihnen deshalb möglich, das Ziel eines stabilen Gesundheitssystems konsequent zu verfolgen; ein Ziel, das aber dem kollektiven Interesse (intérêt public) entspricht. Aus diesem Grund beschloss der Tessiner RR 1998, im Rahmen der NPM-Verwaltungsreform «Amministrazione 2000» ein Projekt auszuarbeiten, um die Rahmenbedingungen für einen richtigen Einsatz der Gesundheitsres- sourcen zu schaffen [4]. Stützpfeiler des Projekts sollen die kulturellen und sozialen Aspekte und ihre Auswirkungen auf den Gesundheitsbereich, die Koordination sämtlicher Akteure des Gesundheitswesens, die finanziellen Anreize, die technologische Verbreitung sowie die Planung sein.

Im folgenden Reifeprozess konzentrierte sich das Projekt «Rete sanitaria» (Gesundheitsnetz) gerade auf die technologische Verbreitung sowie auf die kulturellen und sozialen Komponente. Das Projekt soll alle Gesundheitsakteure anspornen, mittel- bis langfristige Strategien zu entwickeln, welche zur Verbesserung der Koordination und der Zusammenarbeit im Gesundheitssystem beitragen. Denn letzteres erweist sich einerseits gewiss als flächendeckend und grosszügig, doch anderseits auch als komplex, redundant und zersplittert. Ein Projekt also, das gleichzeitig als Zündmittel und Katalysator eines Prozesses kultureller Wandlung wirken soll. Ein Projekt, das von den Hauptakteuren des kantonalen Gesundheitssystems in den kommenden Jahren in die Tat umgesetzt werden soll.

Die modernen Informationstechnologien stellen in dieser Hinsicht - gerade weil sie bezüglich der Machtverhältnisse im Gesundheitswesen neutral sind - die beste Strategie dar, um die gesetzten Ziele in die Tat umzusetzen. Angesichts der Komplexität und Zersplitterung des Systems erscheint ein effizienterer Informationsaustausch zwischen allen Beteiligten unabdingbar. Womit sich nicht nur die Effizienz und Qualität, sondern auch die Sicherheit (Datenschutz) des Systems verbessern sollten.

Verschiedene E-Health-Care-Instrumente wurden dabei geprüft: von der Telemedizin zur elektronischen Krankengeschichte, von der Gesundheitskarte zum elektronischen Gesundheitsnetz, wobei jedes Instrument nach Durchführbarkeit und Zweckmässigkeit geprüft worden ist. Nach einem Prozess gründlicher Vertiefung konzentrierte sich die Steuerungsgruppe schliesslich auf das Instrument der elektronischen Gesundheitskarte. Auf den ersten Blick unscheinbar - und somit emotionell neutral bietet die Gesundheitskarte unermessliche Möglichkeiten, um die Gewohnheiten von Patienten 
und Leistungserbringer radikal umzustürzen. Sowohl als Mittel zur Datenübertragung (auf einem Mikrochip) als auch als elektronischer Schlüssel zum Netz, wird die Karte nicht nur zum reinen Steuerungs- und Harmonisierungsmittel im neuen Informatiknetz, sondern stellt darüber hinaus den Patienten in den Mittelpunkt des ganzen Gesundheitssystems. Denn die Karte soll den Patienten zur vermehrten Selbstbestimmung (Empowerment) und zu einer besseren Verantwortung für seine Gesundheit anregen: Darin liegt der revolutionäre Charakter der elektronischen Gesundheitskarte.

Nicht nur in den meisten europäischen Ländern (allen voran Deutschland, Frankreich und Italien) können solche Erfahrungen bereits erfolgreich bestätigt werden, sondern auch in Kanada, den Vereinigten Staaten und Japan, wo die Karte nicht nur dazu dient, das administrative Prozedere zu vereinfachen (Versichertenkarte), sondern auch die Qualität der Behandlungen zu verbessern (Gesundheitskarte). Im März 2002 einigten sich die EU-Staaten im Rahmen ihrer jeweiligen Arbeitspolitik auf die Einführung einer obligatorischen Versichertenkarte.

Kraft der bilateralen Verträge sieht sich auch die Schweiz dazu gezwungen, den selben Schritt zu unternehmen, das heisst eine eurokompatible Versichertenkarte einzuführen, was im Rahmen der zweiten KVG-Revision (zur Debatte bei der Bundesversammlung) geschieht. Da jedoch die Gesundheitspolitik nicht Sache des Bundes ist, sondern der kantonalen Zuständigkeit unterliegt, kann die Entwicklung eines solchen Instruments, dessen Einsatz über den buchhalterischen und versicherungstechnischen hinausgehen soll, nur eben auf kantonaler Ebene erfolgen. Denn bei der Gesundheitskarte handelt es sich um ein Steuerungsinstrument des gesamten Gesundheitswesens, währenddem die Funktion als Instrument der Krankenversicherung bloss ein Teilaspekt davon ist. Der Bund erkannte diese Problematik und lud den Kanton Tessin - dessen Projekt in der Botschaft über die Versichertenkarte ausdrücklich erwähnt ist (Art. 42 a) aufgrund seiner Erfahrung dazu ein, bei der Entwicklung der «KVG-Karte» mitzuwirken.

Auf der zukünftigen Gesundheitskarte, welche internationale Standards erfüllen muss, kann der Patient beispielsweise Notfalldaten, Allergien, Impfungen, die eingenommenen Medikamente oder die wichtigsten Angaben zu seiner Krankengeschichte eingeben. Dank des Zugriffs auf solche Informationen durch die Fachpersonen kann dem Patienten unter Umständen das Leben gerettet werden und unnötiges Leiden erspart bleiben. Gleichzeitig können dabei auch die Kosten eingespart werden. Ausserdem dürfte die Karte, als Zugriffsschlüssel auf das elektronische Gesundheitsnetz, noch grössere Erfolge verbuchen. Die Einführung der Karte parallel zur Entwicklung des elektronischen Netzes garantiert einen sicheren Informationsaustausch zwischen den verschiedenen an der Gesundheitsversorgung beteiligten Akteuren. Damit sollte sich das elektronische Gesundheitsnetz harmonisch rund um die Karte - als verbindendes Element - entwickeln können.

Die Aussicht, dass sich Gesundheitskarte und elektronische Vernetzung gegenseitig ergänzen mögen, hat sich im August 2002 mit der Unterzeichnung einer Zusammenarbeitserklärung zwischen den Gesundheitsdepartementen Tessin und Genf konkretisiert. Der Kanton Genf ist zurzeit dabei, die Merkmale eines gemeinsamen elektronischen Gesundheitsnetzes zu definieren, auf welches über einen Schlüssel (beispielsweise die «Gesundheitskarte») - unter Wahrung der Rechte des Patienten - zugegriffen werden kann.

Bei den beiden Projekten spielt die technologische Komponente allerdings eine untergeordnete Rolle. Die wahre Herausforderung liegt im kulturellen Bereich: Heute wird im Gesundheitswesen die Informatik als Hilfsmittel bei der Patientenbetreuung noch sehr begrenzt angewendet. Aus diesem Grund sind auch ihre Vorund Nachteile noch wenig bekannt, was bei der Bevölkerung leicht Unverständnis und Vorurteile hervorruft, gerade was etwa den Datenschutz betrifft. In diesem Zusammenhang sei allerdings erwähnt, dass es auch bei den herkömmlichen Datenübermittlungssystemen noch zahlreiche Probleme zu lösen gilt. Patienteninformationen, die via Fax oder per Post übermittelt werden, können beispielsweise von jeder beliebigen Person gelesen werden. Das gleiche gilt für den häufig ungeschützten Zugang zu Archiven in Spitälern und anderen Einrichtungen. Bei der Gesundheitskarte und der elektronischen Vernetzung der Fachpersonen hingegen entscheidet allein der Patient, welche Daten er wem zugänglich machen will.

Aus dem Banksektor (z. B. EC-Karte) haben wir gelernt, dass die Einführung eines neuen Arbeitsinstrumentes einen langen Lern- und Anpassungsprozess benötigt. Gerade daher wurde im Konsens mit den Gesundheitspartnern ein Pilotversuch gestartet, der nicht nur den Kulturwandel, sondern auch die operativen Schritte erleichtern soll. Nach einer 18monatigen Vorbereitungsphase, während der die Partner eruiert, die Karten produziert und das im Gesundheitswesen tätige Personal geschult werden soll, wird die Gesundheitskarte in der städti- 
Rund 3000 - 4000 Patienten und 300 - 400 Fachpersonen, darunte Ärzte, Apotheker, öffentliche und private Spitäler, Notfalldienste und Spitex sollen am Pilotprojekt teilnehmen. schen Umgebung von Lugano versuchsweise eingeführt. * Parallel dazu werden verschiedene Informationskampagnen durchgeführt werden. Nach der Pilotphase wird es möglich sein, dank einer erhöhten Akzeptanz der Bevölkerung die nötigen Gesetzesänderungen vorzuschlagen (kantonale Gesundheitsgesetz), um schliesslich die Benützung der Karte auf den ganzen Kanton auszudehnen und gleichzeitig die Schaffung eines homogenen elektronischen Gesundheitsnetzes voranzutreiben. Eine pragmatische und zeitaufwendige Strategie, welche es dem Staat aber anderseits erlaubt, den Bedürfnissen der Bevölkerung unter Wahrung ihrer Rechte nachzukommen und gleichzeitig einen Konsens der Akteure zu erlangen. Womit die Strategie der für die Schweiz typischen Konsenssuche entspricht.

Dass der Kanton Tessin dabei den richtigen Weg beschritten hat, beweisen die zahlreichen Anerkennungen von aussenstehenden Beobach- tern. In erster Linie vom Eidgenössischen Departement des Innern (im besonderen vom BSV), welches das Tessiner Projekt mit besonderem Interesse mitverfolgt. Aber auch andere Kantone und verschiedene Berufsverbände haben ihr Interesse am Projekt kundgetan. Auch das Bundesamt für Berufsbildung und Technologie (Eidgenössisches Volkswirtschaftsdepartement) hat dem Projekt ausdrücklich innovative Werte, Pioniergeist und die Fähigkeit, den Konsens der Gesundheitsakteure zu erlangen, zuerkannt.

Durch seinen übergreifenden Ansatz bereichert und ergänzt somit das Projekt des Kantons Tessin verschiedene Initiativen im Bereich des Angebots und der Nachfrage im Gesundheitswesen. Angesichts der Schwierigkeiten, denen die Sparmassnahmen im Gesundheitswesen begegnen, wird offensichtlich, dass einzig eine gemischte Strategie einen Hoffnungsschimmer auf Erfolg zulässt.

\section{eHealthCare.ch 03}

Neue Technologien und IT in Medizin und Gesundheitswesen

Konferenz und Fachmesse

Universität Zürich-Irchel, 16./17. Oktober 2003

Schwerpunkt: Der Patient im Informationszeitalter

www.eHealthCare.ch
Leading Mediapartner: EMH Schweizerischer Ärzteverlag AG 